\title{
Ultrafast gain and index dynamics of quantum dash structures emitting at $1.55 \mathrm{mu} \mathrm{m}$
}

Poel, Mike van der; Mørk, Jesper; Somers, A.; Forchel, A.; Reithmaier, J. P.; Eisenstein, G.

Published in:

Applied Physics Letters

Link to article, DOI:

$10.1063 / 1.2337881$

Publication date:

2006

Document Version

Publisher's PDF, also known as Version of record

Link back to DTU Orbit

Citation (APA):

Poel, M. V. D., Mørk, J., Somers, A., Forchel, A., Reithmaier, J. P., \& Eisenstein, G. (2006). Ultrafast gain and index dynamics of quantum dash structures emitting at $1.55 \mathrm{mu}$ m. Applied Physics Letters, 89(8), 081102. https://doi.org/10.1063/1.2337881

\section{General rights}

Copyright and moral rights for the publications made accessible in the public portal are retained by the authors and/or other copyright owners and it is a condition of accessing publications that users recognise and abide by the legal requirements associated with these rights.

- Users may download and print one copy of any publication from the public portal for the purpose of private study or research.

- You may not further distribute the material or use it for any profit-making activity or commercial gain

- You may freely distribute the URL identifying the publication in the public portal 


\title{
Ultrafast gain and index dynamics of quantum dash structures emitting at $1.55 \mu \mathrm{m}$
}

\author{
M. van der Poel ${ }^{\text {a) }}$ and J. Mørk \\ COM.DTU, NanoDTU, Technical University of Denmark, DK-2800 Kgs. Lyngby, Denmark
}

\begin{abstract}
A. Somers and A. Forchel
Technische Physik, Physikalisches Institut, Universität Würzburg, Am Hubland, D-97074 Würzburg, Germany

J. P. Reithmaier

Technische Physik, Institute of Nanostructure Technologies and Analytics, Universität Kassel, Heinrich-Plett-Str. 40, D-34132 Kassel, Germany

G. Eisenstein

Technion, Israel Institute of Technology, Technion City, Haifa 32000, Israel
\end{abstract}

(Received 6 February 2006; accepted 15 July 2006; published online 21 August 2006)

\begin{abstract}
The authors systematically characterize the ultrafast gain and index recovery of a quantum dash semiconductor optical amplifier after it has amplified a strong femtosecond pulse. The results show a recovery dominated by a fast time constant of $1.4 \mathrm{ps}$ with an ultimate recovery taking place on a $150 \mathrm{ps}$ time scale. The results are distinctly different from the recovery of quantum dot amplifiers and reflect the special density of states of the quantum-wire-like dashes. (c) 2006 American Institute of Physics. [DOI: 10.1063/1.2337881]
\end{abstract}

Low-dimensional quantum dash (Qdash) and quantum dot (Qdot) optoelectronic devices have proven to exhibit a number of unique properties allowing for improved performance and different applications compared to bulk and quantum well based devices. The properties of Qdot based devices have been intensely studied. ${ }^{1,2}$ Less work has been done on InAs Qdashes grown on InP substrates, but this system also exhibits promising results ${ }^{3,4}$ at the important wavelength around $1.55 \mu \mathrm{m}$. An important question arises therefore as to which extent zero-dimensional Qdot properties are to be found in Qdash structures, where the gain material is wirelike (elongated dots) and thus quasi-one-dimensional. It was shown by Dery et al. ${ }^{5}$ that the gain properties of a Qdash amplifier bear the distinct fingerprint of a quantum-wire-like density of states.

In this letter we present results of ultrafast pump-probe spectroscopy on a Qdash amplifier operating in the $1.55 \mu \mathrm{m}$ region. This type of experiment, where gain and index changes of a Qdash semiconductor optical amplifier (SOA) are measured with high time resolution, is particularly useful in assessing the dynamical processes and characteristic times associated with carrier relaxation and gain recovery. Such information sheds light on the ultimate device limitations dictated by the dynamics of Qdash gain materials.

The device under investigation is a $1.4 \mathrm{~mm}$ long singlemode ridge-waveguide SOA with an active layer consisting of four sheets of self-assembled InAs/InGaAlAs Qdashes. ${ }^{3}$ Antireflection coatings of the device facets reduce feedback to a level below $0.1 \%$ and thus inhibit lasing. The amplified spontaneous emission (ASE) spectrum shows ground-state emission in the $1550 \mathrm{~nm}$ range (see inset of Fig. 1). The ASE spectrum of a Qdash SOA comparable to the one studied in this letter was analyzed by Dery et $a .^{5}$ who found that only a quantum-wire-like density of states could provide a quan-

\footnotetext{
${ }^{a)}$ Electronic mail: mvp@com.dtu.dk
}

titative explanation of the observed spectrum and its dependence on injected current. The large inhomogeneous Qdash broadening allow for low cross-talk amplification of wavelength division multiplexed signals. ${ }^{6}$

The small signal modal gain of the device as function of current was measured at 1525, 1560, and $1596 \mathrm{~nm}$ (Fig. 1). Before saturation the differential gain at $1560 \mathrm{~nm}$ is higher than at $1520 \mathrm{~nm}$, reflecting that energetically lower states are responsible for the gain at this longer wavelength. The slow and gradual gain saturation with current rather resembles bulk or shallow InAs/GaAs QD SOAs (Ref. 7) than deeply confined Qdot SOAs, ${ }^{8}$ indicating a strong coupling between the active states in the Qdashes and the surrounding continuum states. In this respect it should be noted that the maximum gain even at high currents is significantly smaller than the maximum absorption at zero bias, indicating a problem of reaching complete inversion of the active states in this structure, which also points to a strong coupling between the active states and the continuum in the barriers.

Gain and phase changes of a weak probe pulse following a stronger pump pulse in the Qdash SOA are measured in a

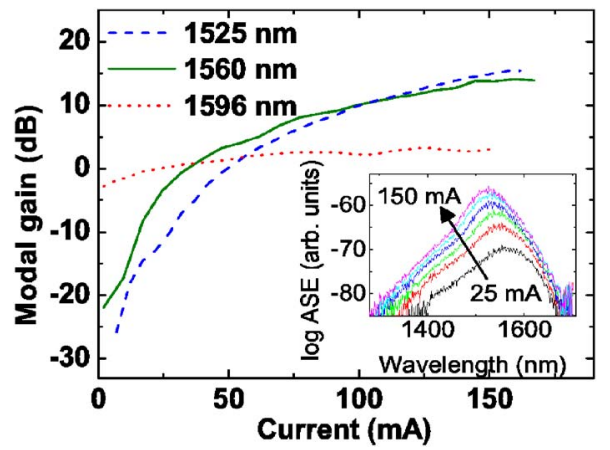

FIG. 1. (Color online) Small signal SOA gain at three different wavelengths. The ASE spectrum of the device at bias current from 25 to $150 \mathrm{~mA}$ in steps of $25 \mathrm{~mA}$ is shown in the inset. 

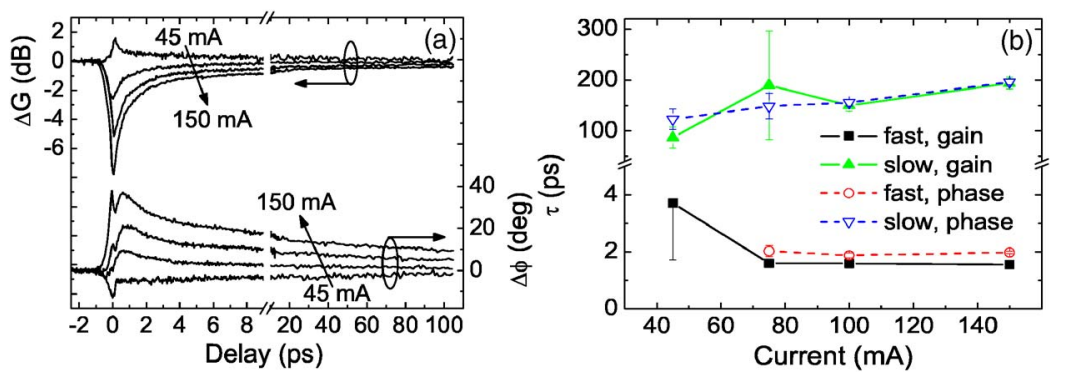

FIG. 2. (Color online) (a) Gain and phase recovery of Qdash SOA for the bias currents of 45, 75, 100, and $150 \mathrm{~mA}$ at a fixed wavelength of $1523 \mathrm{~nm}$. (b) Fast and slow recovery times from biexponential fit to gain and phase recovery traces. degenerate, heterodyne pump-probe setup with pump-probe pulse length of 150 fs (see Ref. 9 for the method and Ref. 10 for details of the setup).

Figure 2(a), left axis, shows the measured gain dynamics. The device was probed at $1523 \mathrm{~nm}$ which corresponds to the gain maximum at $150 \mathrm{~mA}$ bias current. The Qdash recovery proceeds in two distinctly different steps: first a fast component with a characteristic time of ca. $1.6 \mathrm{ps,} \mathrm{which} \mathrm{is}$ responsible for the majority of the recovery, and second a slower component with a characteristic time of ca. 130 ps. The fast component is ascribed to a combination of carrier relaxation where occupied, higher-lying dash carriers relax into the active states and carrier capture from barrier states into the dash. The slow component is ascribed to the recovery of the total carrier density of the device, i.e., determined by the effective carrier lifetime of the component. This picture fits well with the results of Ref. 11. The interpretation is further supported by the fact that at longer wavelengths the gain recovers nearly completely through the fast component (Fig. 3), since the states responsible for amplification at the longer wavelengths are placed relatively close to the band edge and thus only influenced little by carrier density variation at this current. A generally striking feature of the gain recovery in Fig. 2 is the large magnitude of the fast component compared to the slow component, which resembles the behavior seen in Qdot devices. ${ }^{8,12}$

The recovery time constants of the Qdash component in the gain regime do not show any significant dependence on bias current, as shown in Fig. 2(b). This constancy of relaxation times suggests that phonon scattering (see, e.g., Ref. 13) may be the dominating mechanism for dash relaxation and capture also in the Qdash case. ${ }^{12}$ A small variation of the

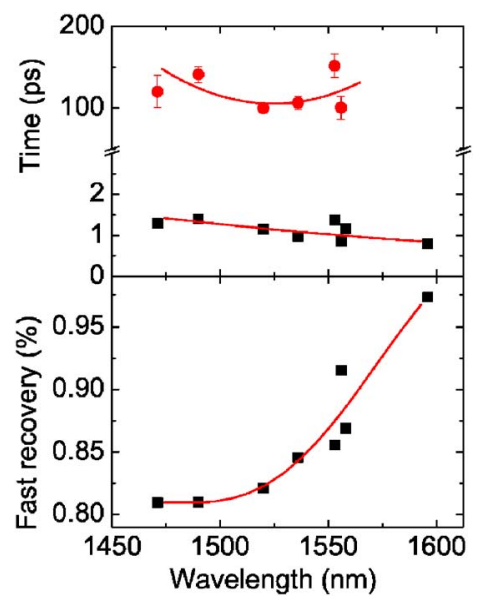

FIG. 3. (Color online) Characteristic gain recovery times of Qdash SOA (upper panel) as function of wavelength and relative amplitude of fast component to total gain recovery as function of wavelength in SOA (lower

panel). Current is fixed at $150 \mathrm{~mA}$. The lines are guides to the eyes. pump-probe delay. Lower panel: Phase recovery.
Downloaded 17 Jun 2010 to 192.38 .67 .112 . Redistribution subject to AlP license or copyright; see http://apl.aip.o
Qdash SOA recovery times was seen with wavelength (Fig. 3). This behavior reflects the large inhomogeneous broadening of the Qdash ensemble probed in the experiment and is similar to what has been observed in Qdots. ${ }^{12}$

The results of Figs. 2 and 3 also indicate an effective carrier lifetime, which is longer than what is seen in typical bulk SOAs of the same length, which is most likely caused by the smaller modal gain in the Qdash component. The lower gain thus results in less ASE per unit length and consequently in a longer stimulated emission time of the carriers even in a long device. However, the nature of the continuum states of Qdash, from which carriers are fed into the active states, is not yet well understood, and further material engineering may strongly affect the time constant.

The phase dynamics plotted in Fig. 2(a) is more complex than the corresponding gain changes. Firstly we note an instantaneous component occurring only when the pump and probe are arriving at the same time. Such instantaneous index nonlinearities are ascribed to the Stark and Kerr effects. ${ }^{14}$ After this initial spike in the phase trace, we see carrier cooling effect as a slow phase increase until ca. 2 ps. The long-time phase recovery is given by an exponential decay with the same characteristic time scale as the corresponding gain recovery [see Fig. 2(b)]. This behavior suggests that the carrier distribution is in quasiequilibrium as the carrier density recovers to its original value. The slow recovery is much more dominating in the phase case compared to the gain recovery. This difference in the behavior of the gain and the phase is consistent with the fact that the refractive index is strongly affected by changes of the population of the states in neighboring dashes, any potential wetting layer, and/or barrier regions, whereas only the population of the active states affects the gain.

Figure 4 shows comparison of the gain and index recovery traces to a reference Qdot SOA with an active layer of

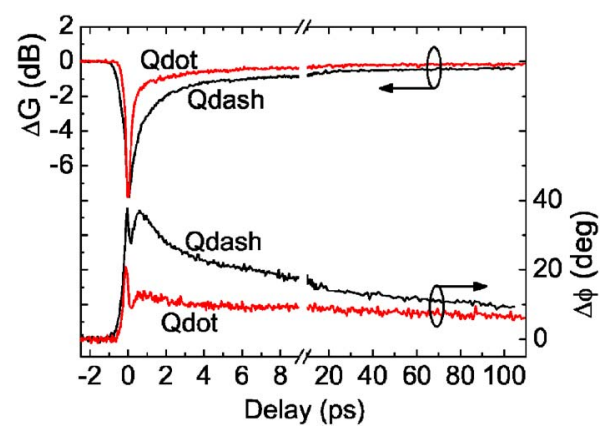

FIG. 4. (Color online) Comparison of Qdash and Qdot SOA pump-probe traces. Both devices are electrically pumped to be high in the gain regime and they are probed at their respective gain maxima. Upper panel: Gain recovery. Qdot trace has been scaled to same gain compression at zero pump-probe delay. Lower panel: Phase recovery. 
self-organized InAs/InGaAs/GaAs Qdots. ${ }^{12,15}$ Both devices are biased well above the transparency point and pumped and probed at their respective gain maxima which are $1523 \mathrm{~nm}$ (Qdash) and $1260 \mathrm{~nm}$ (Qdot). The Qdot component is seen to have an initial recovery much faster than the Qdash component. The very fast Qdot SOA recovery stems from phonon-mediated carrier relaxation in the dots which efficiently fills the depleted Qdot states and thereby closes the spectral hole burned by the strong pump pulse. ${ }^{12}$ On an intermediate time scale of ca. $4 \mathrm{ps}$, the Qdot recovery consists of a refilling of the dot by capture from the wetting layer. Ultimately, the Qdot component recovers on an $80 \mathrm{ps}$ time scale. The Qdot gain recovery trace may thus only be fitted with a triexponential function as opposed to the Qdash trace which is well fitted with a biexponential. Qdots display discrete energy levels and have only few states available for carriers. The Qdashes, on the other hand, have a continuum of states and no well-defined wetting layer. For this reason, one may tentatively suggest that the fast 1.6 ps recovery time seen in the Qdash component reflects that the intradash relaxation and capture processes are mixed together.

Bulk devices may show a very fast component, which in that case is interpreted as a combination of spectral hole burning and two-photon absorption. ${ }^{14}$ However, in that case, the recovery time is governed by the carrier-carrier scattering time of the order of 50-100 fs, whereas here it is more than a picosecond long. Together with the Qdash size quantization in one dimension, which leads to a quantum-wire-like density of states, these features allow for a much more effective spectral hole burning compared to bulk devices. The effect may be utilized in devices for all-optical signal processing, although this requires more characterization and a better understanding of the dynamics on the long time scale, when the device responds to a long train of short pulses. ${ }^{11}$

The investigated Qdash components show a much larger phase response compared to the Qdot component. This suggests a larger linewidth enhancement factor (LEF) of this component compared to the Qdot SOA. This behavior is in agreement with expectations from the tail toward high energy in the quantum-wire density of states. A large LEF leads to chirp in the amplified pulse and limits the component's use in high speed systems. It is, however, well known that the Qdot LEF depends strongly on the detailed energy level structure and may show values ranging from approximately zero to values comparable to that found in quantum well based devices, and such a variation would also be expected from Qdash based components with different energy structures.

This work was financially through the IST project BigBand.

${ }^{1}$ M. Sugawara, N. Hatori, M. Ishida, H. Ebe, Y. Arakawa, T. Akiyama, K. Otsubo, T. Yamamoto, and Y. Nakata, J. Phys. D 38, 2126 (2005).

${ }^{2}$ D. Bimberg and C. Ribbat, Microelectron. J. 34, 323 (2003).

${ }^{3}$ R. Schwertberger, D. Gold, J. P. Reithmaier, and A. Forchel, IEEE Photonics Technol. Lett. 14, 735 (2002).

${ }^{4}$ R. H. Wang, A. Stintz, P. M. Varangis, T. C. Newell, H. Li, K. J. Malloy, and L. F. Lester, IEEE Photonics Technol. Lett. 13, 767 (2001).

${ }^{5}$ H. Dery, E. Benisty, A. Epstein, R. Alizon, V. Mikhelashvili, G. Eisenstein, R. Schwertberger, D. Gold, J. P. Reithmaier, and A. Forchel, J. Appl. Phys. 95, 6103 (2004).

${ }^{6}$ R. Alizon, D. Hadass, V. Mikhelashvili, G. Eisenstein, R. Schwertberger, A. Somers, J. P. Reithmaier, A. Forchel, M. Calligaro, S. Bansropun, and M. Krakowski, Electron. Lett. 40, 760 (2004).

${ }^{7}$ D. R. Matthews, H. D. Summers, P. M. Smowton, and M. Hopkinson, Appl. Phys. Lett. 81, 4904 (2002).

${ }^{8}$ P. Borri, W. Langbein, J. M. Hvam, F. Heinrichsdorff, M.-H. Mao, and D. Bimberg, IEEE Photonics Technol. Lett. 12, 594 (2000).

${ }^{9}$ A. Mecozzi and J. Mørk, J. Opt. Soc. Am. B 13, 2437 (1996).

${ }^{10}$ P. Borri, W. Langbein, J. Mørk, and J. M. Hvam, Opt. Commun. 169, 317 (1999).

${ }^{11}$ T. W. Berg, S. Bischoff, I. Magnusdottir, and J. Mørk, IEEE Photonics Technol. Lett. 13, 541 (2001).

${ }^{12}$ M. van der Poel, E. Gehrig, O. Hess, D. Birkedal, and J. M. Hvam, IEEE J. Quantum Electron. 41, 1115 (2005).

${ }^{13}$ I. Magnusdottir, A. V. Uskov, S. Bischoff, B. Tromborg, and J. Mørk, J. Appl. Phys. 92, 5982 (2002).

${ }^{14}$ J. Mørk and A. Mecozzi, J. Opt. Soc. Am. B 13, 1803 (1996).

${ }^{15}$ M. V. Maximov, A. F. Tsatsul'nikov, B. V. Volovik, D. S. Sizov, Yu. M. Shernyakov, I. N. Kaiander, A. E. Zhukov, A. R. Kovsh, S. S. Mikhrin, V. M. Ustinov, Zh. I. Alferov, R. Heitz, V. A. Shchukin, N. N. Ledentsov, D. Bimberg, Yu. G. Musikhin, and W. Neumann, Phys. Rev. B 62, 16671 (2000). 